\title{
Factors associated with maternal delays in utilising emergency obstetric care in Arsi Zone, Ethiopia
}

\author{
Y Wondu, ${ }^{1} \mathrm{MSc}$; B Dibaba, ${ }^{2} \mathrm{MPh}$; M Bayu, ${ }^{3} \mathrm{MSc}$; M Hussien, ${ }^{4} \mathrm{MSc}$ \\ ${ }^{1}$ Department of Midwifery, College of Health Science, Arsi University, Asella, Ethiopia \\ ${ }^{2}$ Department of Public Health, College of Health Science, Arsi University, Asella, Ethiopia \\ ${ }^{3}$ Department of Nursing, College of Health Science, Arsi University, Asella, Ethiopia \\ ${ }^{4}$ Department of Pharmacy, College of Health Science, Arsi University, Asella, Ethiopia
}

Corresponding author: $Y$ Wondu (wonyir@gmail.com)

\begin{abstract}
Background. Delay to timely healthcare contributes to high maternal mortality and morbidity in developing countries. The so-called 'Three delays' model has been used extensively to investigate factors relating to maternal mortality.

Objective. To investigate factors associated with delayed emergency obstetric care in Arsi Zone, Ethiopia.

Methods. A cross-sectional study was conducted across 10 public health facilities in Arsi Zone, Ethiopia. The required sample size was calculated as 847 , with the number of participants required at each facility determined proportionally.

Results. Data from 775 respondents were used in the analysis. Approximately a quarter of respondents $(n=203 ; 26.2 \%)$ reported a delayed decision to seek emergency obstetric care. The mean time for delay was 90 minutes (range 30 minutes - 18 hours). Maternal age, educational level, monthly household income and antenatal visits were significant predictors of this first maternal delay. Close to a third of the respondents $(n=234 ; 30.2 \%)$ reported a transport-related delay in reaching a healthcare facility; some respondents walked at least 30 minutes to reach the facility. Approximately a quarter of respondents $(n=198 ; 25.5 \%)$ reported that they did not receive timely care after arriving at the healthcare facility. The mean delay was 42.3 minutes.

Conclusion. The most common delay was related to difficulty in reaching the healthcare facility. In approximately half of the cases, the woman's husband took the decision to access medical care. This suggests limited independent decision-making power of women in this context. Such factors should be considered in efforts to reduce maternal morbidity and mortality.
\end{abstract}

S Afr J Obstet Gynaecol 2019;25(2):56-63. https://doi.org/10.7196/SAJOG.2019.v25i2.1437

Maternal deaths due to pregnancy-related complications pose a continuing healthcare challenge in developing countries, ${ }^{[1,2]}$ with maternal mortality having been identified as a priority in health policy and research agendas. ${ }^{[3,4]}$ In many low- and middle-income countries, mortality rates related to pregnancy are high and have a considerable negative effect on the population of reproductive-age women. These deaths can mostly be avoided by timely and adequate treatment. ${ }^{[5]}$

The 'Three delays' model, developed in the 1990s as a way to understand factors that may contribute to maternal mortality, describes three categories of delays in maternal care and has been used throughout the world to understand and reduce maternal mortality, especially in low- and middle-income countries. ${ }^{[6,7]}$

The first delay occurs at the level of the mother, family or community when a life-threatening condition is not recognised. Most deaths occur during labour or in the first 24 hours post partum. In many cases, births take place at home and without assistance of skilled attendants who can recognise and respond to an emergency in time to prevent adverse outcomes. ${ }^{[8-10]}$

The second delay is associated with reaching a healthcare facility and may be due to poor road conditions, lack of transportation or a remote location. Poorly constructed roads and lack of access to vehicles are the main hurdles in accessing healthcare facilities in time. Animal-drawn transport is often the most readily available form of transport. ${ }^{[8,10,11]}$
The third delay occurs at the healthcare facility. This delay can involve a delay in (i) receiving appropriate care at the initial facility, (ii) transferring a patient to another facility for definitive care if needed, and (iii) ensuring that appropriate care is provided at the second facility. Delays at any of these points have been shown to worsen patient outcome. ${ }^{[8,10,12]}$

In many developing countries, women and their families face socioeconomic and cultural barriers to seeking professional care, including having to travel long distances to healthcare facilities, a lack of knowledge about danger signs during pregnancy and the practice of using untrained local practitioners during delivery. ${ }^{[13,14]}$ In Ethiopia, providing timely treatment for obstetric emergencies and increasing women's access to and use of facilities for childbirth form part of a critical national strategy to improve maternal health outcomes. ${ }^{[15]}$ The Averting Maternal Deaths and Disability (AMDD) programme, which is active in many developing countries, is a collaboration between Columbia University in the USA and the United Nations. The programme uses a supply-side approach to support the existing capacity of a system and so increase the capacity of an institution for providing basic and comprehensive emergency obstetric care to all women. The aim is to reduce delays in receiving adequate care when a facility is reached. ${ }^{[16]}$

According to recent data from 171 countries, maternal mortality has substantially declined between 1990 and 2015, but progress has been much slower than required to meet the target 
of a $75 \%$ reduction as described in the fifth Millennium Development Goal..$^{[9]}$ It has been estimated that $74 \%$ of maternal deaths can be averted if all women received appropriate emergency obstetric care, ${ }^{[11]}$ specifically as poor quality of care may contribute to an undesirable outcome. ${ }^{[17]}$

This study investigated factors affecting the delivery of maternal care, as described by the 'Three delays' model (Fig. 1), in Arsi Zone, Ethiopia.

\section{Methods \\ Study area}

The study was conducted at selected public healthcare facilities in Arsi Zone, Ethiopia, between 15 October and 30 December 2016. Arsi is located in the Oromia region in south-east Ethiopia, with Asella as its capital town. The area is located at an altitude of $2247 \mathrm{~m}$ above sea level. It is $175 \mathrm{~km}$ from Addis Ababa (the capital of Ethiopia). The zone has a total population of 3280150 , of whom 725890 are of childbearing age as confirmed by the zonal health office in 2015. The majority of inhabitants belong to the Oromo ethnic group. Major occupations include farming and civil service.

\section{Study design and population}

This was a cross-sectional study based on a quantitative method. All women admitted for emergency obstetric care (including pregnant women, those in labour or postnatal mothers) at the selected healthcare facilities during the study period were eligible for participation. Participants had to be willing to consent to individual interviews.

Women who were not conscious or unable to communicate, those who had been readmitted after an earlier inclusion and those who were transferred to another facility after earlier inclusion, were excluded from the study.

\section{Sampling}

\section{Sample size}

The sample size was determined according to the following formula:

$$
N=\frac{Z^{2} P(1-P)}{d^{2}}
$$

where:

$N=$ sample size

$Z$ = standard normal distribution, set at 1.96

$d=$ margin of error in the study, set at 0.05
$P=$ percentage of women who encountered any level of delay (set at 50\%, as there has been no previous study of this kind in the area).

This yielded a sample size of $N=384$. However, considering the design effect, the final sample size was calculated as $N=847((384 \times 2)+79)$, to allow for an approximate non-response rate of $10 \%$.

\section{Sampling technique}

A multistage sampling technique was used (Fig. 2). This involved randomly selecting one health centre from each of 10 selected districts. To derive the final sample, the calculated sample size $(N=847)$ was proportionally allocated to the selected facilities based on their average number of deliveries over the previous 6 months.

Subjects were randomly selected at each facility using a sampling interval of $K=N_{\text {facility }} / n_{\text {facility }}$, with $N_{\text {facility }}$ being the total number of deliveries during the study period at the selected facility and $n_{\text {facility }}$ being the required sample from that facility.

\section{Data collection and quality}

Data were collected using a questionnaire developed for the local context based on relevant literature. For data collection, 10 field workers were recruited, all with at least diploma-level education in a health profession background and able to communicate in both Afaan Oromo and Amharic. Two days' training was provided, covering the study objective and procedure, confidentiality, respondents' rights and informed consent.

The questionnaire was prepared in English and translated into Afaan Oromo and Amharic, and then back-translated again by speakers fluent in all languages to check for consistency. In addition, a pilot study was conducted among 42 respondents (5\% of the final total required sample size) who lived outside the sampled areas. Comments from senior academics and obstetric specialists were also incorporated to further improve internal validity. The principal investigator and the co-investigators supervised the data collection process. Forms were checked daily for completeness, logical errors and unclear or irrelevant information.

\section{Operational definitions}

The following operational definitions of maternal delays were used:

Delay 1: The delay relates to a client's health-seeking behaviour, based on the respondent's self-report regarding the gap between the onset of symptoms of obstetric problems and deciding on seeking care.

Delay 2: The delay refers to transportrelated problems evidenced by the respondent's self-report of the time from the decision to seek care to arrival at the health facility.

Delay 3: This refers to a respondent's description of any delays in receiving obstetric care after arrival at the health facility. Emergency obstetric care: Emergency

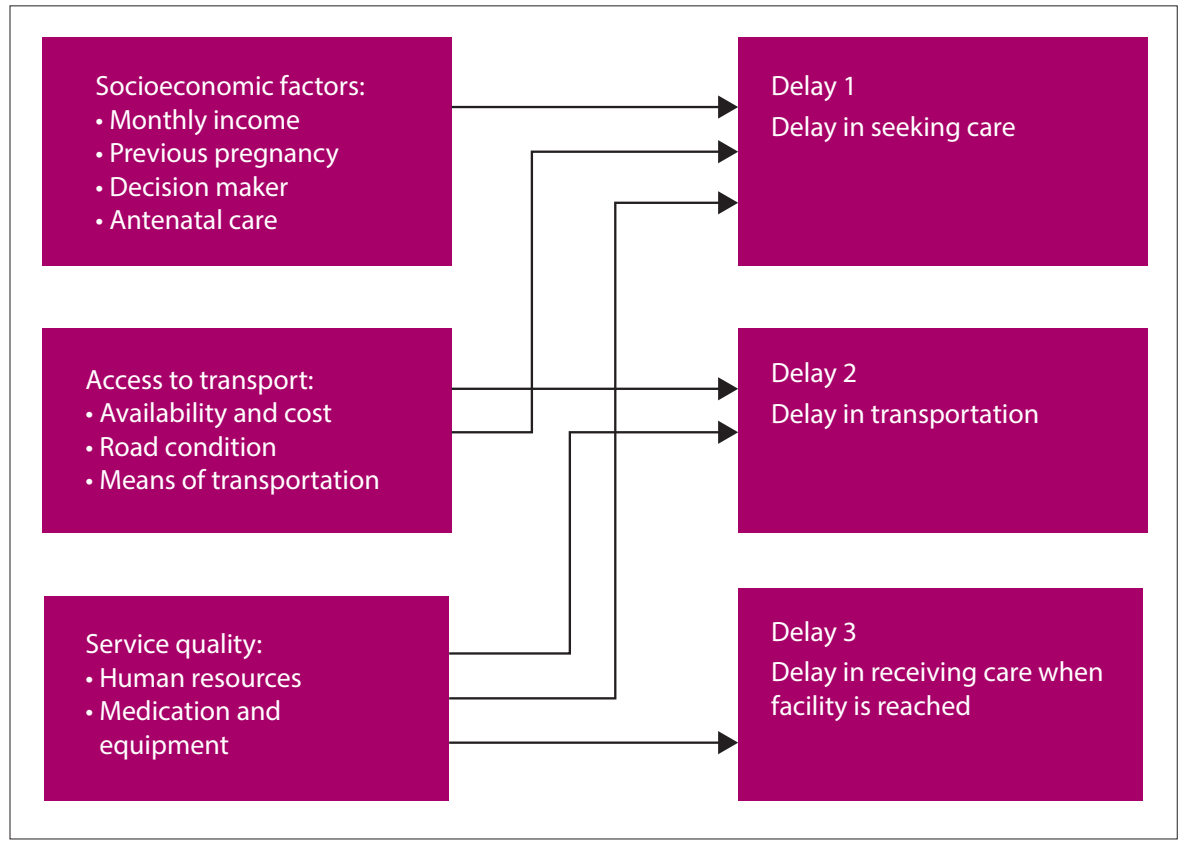

Fig. 1. Conceptual framework showing the relationship of factors with maternal delays, adapted from previous study. 


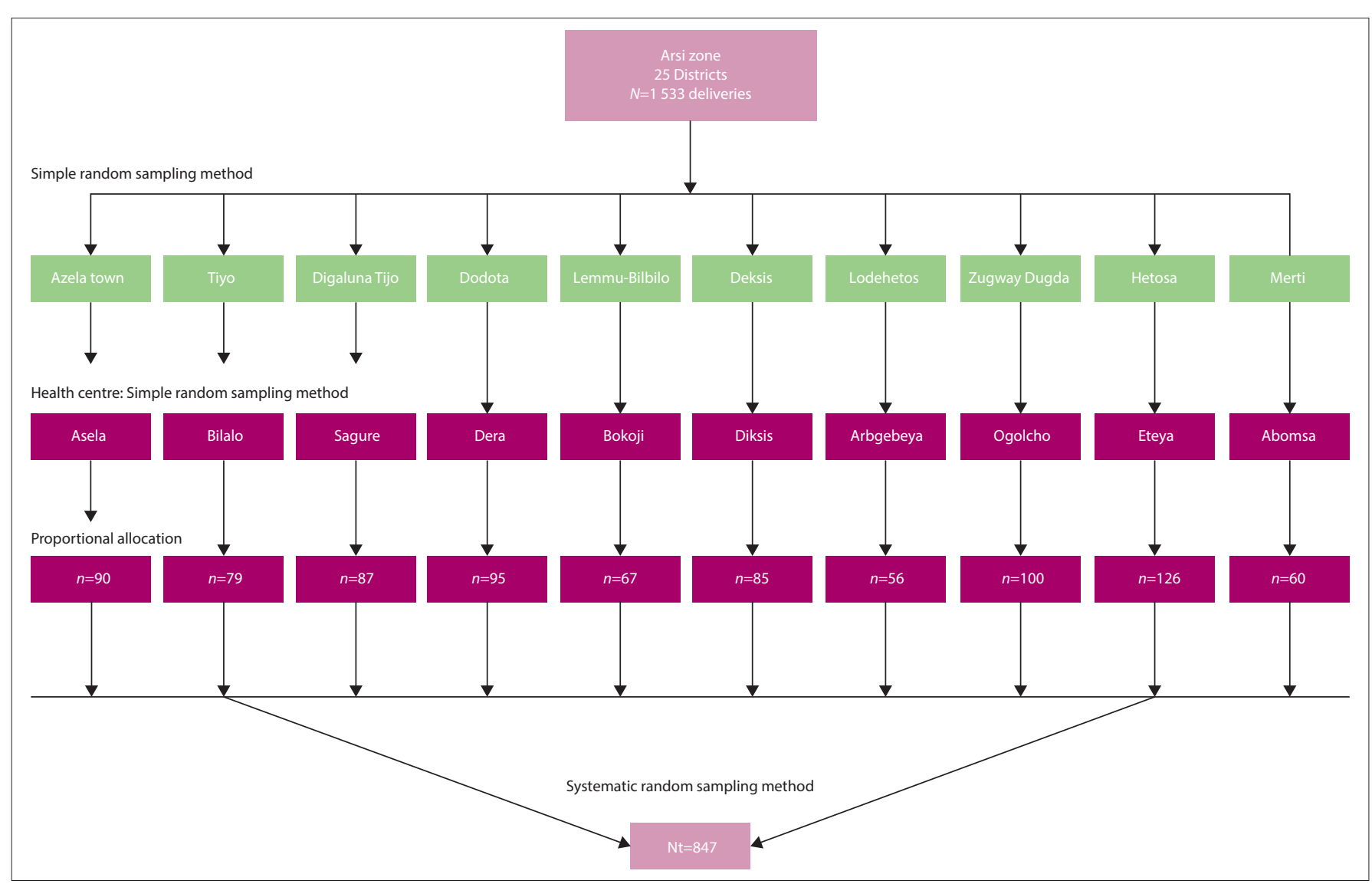

Fig. 2. Schematic representation of sampling procedure. $(N t=$ total final sample; $N=$ number of deliveries. $)$

medical or surgical attention given to a woman who is pregnant, in labour or has recently given birth.

\section{Data analysis}

Epi Info software (version 3.3.2) was used to record collected data, which were then exported to SPSS (version 20) for statistical analysis. Descriptive statistics including percentages, means and standard deviations were calculated. A bivariate logistic regression analysis of each dependent variable (i.e. the three delay categories) was performed against the independent variables, including socioeconomic factors, antenatal care and obstetric history, obstetric consultation and decision-making, means of transport, and the availability of resources (staff, medication or equipment) at the healthcare facility. Variables associated with $p<0.2$ were used in a multivariate regression model to identify independent predictors of maternal delays (outcome variable). A significance level of $p<0.05$ was used.

\section{Ethical considerations}

This study complied with standard protocols for research ethics and was approved by the Ethics and Review committee at Arsi University. The zonal health bureau and the district health office gave permission for the study to be conducted. Informed consent was obtained from all respondents.

\section{Results \\ Sociodemographic characteristics}

Sociodemographic characteristics of the sample are reported in Table 1. Of the 847 women approached for participation in the study, 775 agreed to being interviewed. This translated to a response rate of
$91.5 \%$. The majority of respondents $(n=666 ; 85.9 \%)$ were between 20 and 34 years old; the mean age (and associated standard deviation) was 27.2 (4.9) years. The majority of the respondents were married $(n=685 ; 88.4 \%)$ and 618 respondents $(79.4 \%)$ reported having had up to four previous pregnancies. Just over a quarter $(n=192 ; 28.4 \%)$ of the respondents had completed primary education (higher than Grade 6). Close to half of the respondents $(n=360 ; 46.8 \%)$ indicated associating with the Orthodox Christian religion. Housewives represented the main occupational group $(n=543 ; 70.1 \%)$. Half of the sample $(n=391$; $50.5 \%)$ reported having a monthly family income of ETB500 - 1000 (ZAR1 000 - 2 000). The majority of the respondents $(n=643 ; 83 \%)$ had attended an antenatal clinic during their current pregnancy.

\section{Factors associated with delay in seeking obstetric care (maternal delay 1 )}

Just over a quarter of the respondents $(n=203 ; 26.2 \%)$ reported that they had encountered difficulty in deciding to seek emergency obstetric care. The mean time for delay was estimated at 1.5 hours (range 30 minutes - 18 hours). In $\sim$ half the cases $(n=412 ; 53.2 \%)$, the decision to seek medical care was made by the woman's husband (Fig. 3). More than three-quarters of the respondents $(n=613 ; 79 \%)$ had consulted a traditional birth attendant (TBA) or a health facility before visiting the health centre where they ultimately received obstetric care. For a narrow majority of women ( $n=396 ; 51.1 \%)$, these consultations were at immediately nearby health posts; few had consultations at private clinics $(n=72 ; 9.3 \%)$, other healthcare centres $(n=18 ; 2.3 \%)$ or hospitals $(n=7 ; 0.9 \%)$ (Fig. 4$)$. Less than $20 \%(n=120$; $15.5 \%$ ) had consulted TBAs in their community.

Multivariate logistic regression showed that marital status, parity 
Table 1. Sociodemographic and -economic characteristics of respondents $(N=775)$

\begin{tabular}{ll}
\hline Variable & $\boldsymbol{n}(\%)$ \\
\hline Age & \\
$15-19$ & $40(5.2)$ \\
$20-34$ & $666(85.9)$ \\
35 - 49 & $69(8.9)$ \\
Marital status & \\
Single & $48(6.2)$ \\
Married & $685(88.4)$ \\
Divorced & $30(3.9)$ \\
Widowed & $12(1.5)$ \\
Religious affiliation & \\
Orthodox Christian & $360(46.5)$ \\
Protestant Christian & $156(20.1)$ \\
Catholic Christian & $18(2.3)$ \\
Islam & $241(31.1)$ \\
Occupation & \\
Housewife & $543(70.1)$ \\
Civil servant & $99(12.7)$ \\
Self-employed & $97(12.5)$ \\
Students & $36(4.7)$ \\
Parity & \\
0 & $36(4.6)$ \\
1 - 4 & $618(79.7)$ \\
$\geq 5$ & $121(15.6)$ \\
Educational status & \\
Illiterate & $187(24.1)$ \\
Read and write & $186(24)$ \\
Primary & $192(24.8)$ \\
Secondary & $150(19.4)$ \\
Tertiary & $60(7.7)$ \\
Monthly household income (ETB) & \\
$<500$ & $245(31.6)$ \\
$500-1$ 000 & $393(50.7)$ \\
1 001 - 2 000 & $59(7.6)$ \\
Attendance at antenatal clinic & $78(10.0)$ \\
Yes & \\
No & $643(83.0)$ \\
& \\
& \\
& \\
&
\end{tabular}

and consultation with a TBA or at a health facility before seeking obstetric care were not significantly associated with the first maternal delay (Table 2). However, some categories of all the remaining variables appeared to be associated with a delay in seeking obstetric care.

With regard to age, the delay in seeking care was 2.1 times higher among women between 20 and 34 years of age (adjusted odds ratio (aOR) 2.1; 95\% confidence interval (CI) 1.8 - 5.2) and 4.1 times higher among those older than 35 (aOR 4.1; 95\% CI 2.4 - 10.5) compared with women between 15 and 19 years of age. Not being able to read or write was significantly associated with the delay in deciding to seek obstetric care. The likelihood of the first maternal delay was 5.2 times higher among women who could not read or write (aOR 5.2; 95\% CI 3.4 - 11.9) than among those who had completed tertiary education. The first delay was 3.1 times more likely to present among housewives (aOR 3.1; 95\% CI 1.5 - 7.2) than among students. The odds of delay in seeking obstetric care were

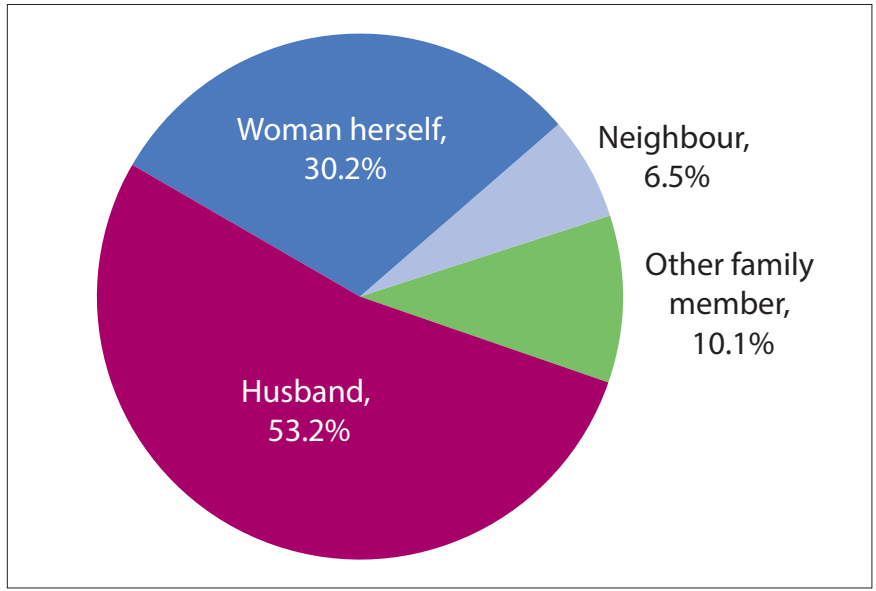

Fig. 3. Distribution of decision-makers for emergency obstetric care.

2.5 times higher among women with a monthly household income $<$ ETB500 (aOR 2.5; 95\% CI 1.5 - 6.3) than among those with a monthly household income $>$ ETB2 000 . The likelihood of the first maternal delay was 4.1 times higher among women who did not attend an antenatal clinic (aOR 4.1; 95\% CI 3.3 - 9.1) than among those who did. The likelihood of a delay in seeking obstetric care was 2.1 times higher if the woman herself had made the decision (aOR 2.1; 95\% CI 1.2 - 3.8) than if the decision was made by family members and neighbours.

\section{Factors associated with delay in reaching emergency obstetric care (maternal delay 2)}

Of the total 775 respondents, 234 (30.2\%) reported that they had experienced a delay in reaching a healthcare facility owing to difficulty in accessing transportation (Table 3). Less than half of the respondents $(n=313 ; 40.4 \%)$ used motorised transport; the remaining respondents walked $(n=282 ; 36.4 \%)$, were carried on a homemade stretcher $(n=116 ; 14.9 \%)$ or used animals as mode of transport $(n=64 ; 8.2 \%)$.

Women older than 19 years were at least 95\% less likely to experience a delay in reaching a healthcare facility owing to difficulty in accessing transportation than women between 15 and 19 years of age (Table 4). Unemployed women were $\sim 9$ times more likely to experience a delay in reaching a healthcare facility ( $\mathrm{aOR}$ 9.3; 95\% CI 3.7 - 23.3) than those who were employed. Delays due to transportation were significantly higher in two subcategories of income compared with women with a monthly household income >ETB2 000 (Table 4).

Women who did not attend an antenatal clinic during their current pregnancy were about twice as likely to experience a delay in reaching a healthcare facility (aOR 1.99; $95 \%$ CI 1.2 - 3.1) as those who did attend an antenatal clinic earlier.

\section{Factors associated with delays in service delivery at the healthcare facility (maternal delay 3)}

Approximately a quarter of respondents ( $n=198 ; 25.5 \%)$ reported experiencing a delay in receiving emergency care at a healthcare facility. The mean delay was calculated as 42 minutes (range 10 minutes - 9 hours). Of the women whose babies were born during spontaneous vaginal delivery, 136 (68.6\%) experienced a delay in receiving emergency care (Table 5). In contrast, among women who reported an abortion, vacuum- 


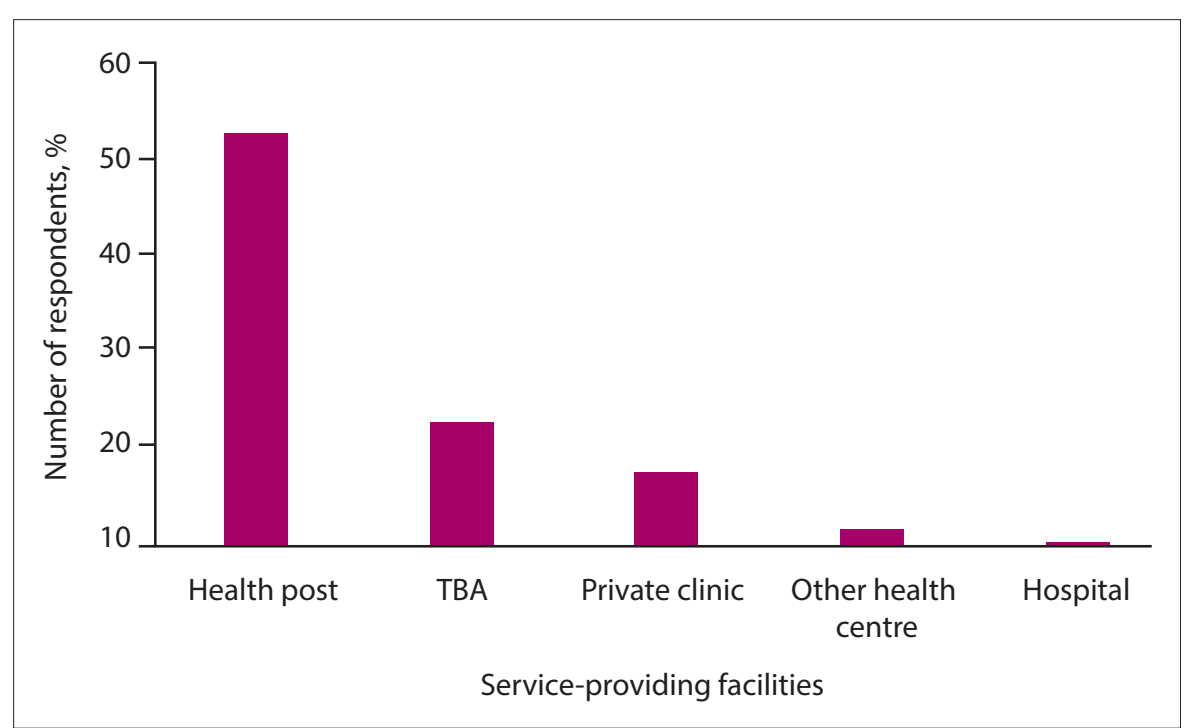

Fig. 4. Services consulted prior to presenting at a healthcare facility for emergency obstetric care. (TBA = traditional birth assistant. $)$ assisted delivery or other delivery assistance, 10,24 and 28 , respectively, reported experiencing a delay in receiving emergency obstetric care at the facility. Notable reasons for the delay included staff workload $(n=90)$ and a careless attitude $(n=36)$ (Table 6).

A mean delay of 42.3 minutes was reported in this category. Multivariate logistic regression revealed significant associations for all variables except marital status and monthly household income. In both the two younger age categories (15 19 years and 20 - 34 years), women were between three and four times more likely to experience this type of maternal delay than women older than 35 years (Table 7). Women who had not attended an antenatal clinic were about three times more likely to experience a delay in service delivery at

Table 2. Multivariate analysis showing factors associated with a delay in seeking obstetric care (maternal delay 1 ) among respondents $(N=775)$

\begin{tabular}{|c|c|c|c|c|}
\hline Variables & No delay experienced, $n$ & Delay experienced, $n$ & OR $(95 \% \mathrm{CI})$ & aOR $(95 \% \mathrm{CI})$ \\
\hline \multicolumn{5}{|l|}{ Age (years) } \\
\hline $15-19$ & 21 & 19 & 1 & 1 \\
\hline $20-34$ & 500 & 176 & $1.9(1.1-2.3)$ & $2.1(1.8-5.2)^{*}$ \\
\hline $35-49$ & 51 & 18 & $2.5(1.7-5.6)$ & $4.1(2.4-10.5)^{*}$ \\
\hline \multicolumn{5}{|l|}{ Marital status } \\
\hline Single & 3 & 45 & $2.9(1.5-4.2)$ & $09(0.6-7.2)$ \\
\hline Married & 571 & 128 & $2(0.9-11.3)$ & $3.6(1.4-12.3)$ \\
\hline Divorced & 3 & 27 & $1.3(1.0-2.1)$ & $2.3(0.9-6.2)$ \\
\hline Widowed & 9 & 3 & 1 & 1 \\
\hline \multicolumn{5}{|l|}{ Educational status } \\
\hline Illiterate & 152 & 35 & $3.8(2.1-5.4)$ & $5.2(3.4-11.9)^{*}$ \\
\hline Only read and write & 128 & 58 & $1.7(0.8-3.4)$ & $2(1.0-13.7)$ \\
\hline Primary education & 138 & 54 & $1.3(0.4-5.0)$ & $2(0.9-7.5)$ \\
\hline Secondary education & 100 & 50 & $0.7(0.8-1.4)$ & $1.2(1.1-6.7)$ \\
\hline Tertiary education & 54 & 6 & 1 & 1 \\
\hline \multicolumn{5}{|l|}{ Occupation } \\
\hline Housewife & 438 & 119 & $5.3(3.1-11.9)$ & $3.1(1.5-7.2)^{*}$ \\
\hline Government employee & 77 & 30 & $2.2(1.8-5.0)$ & $2.1(0.9-3.8)$ \\
\hline Self-employed & 65 & 36 & $3.0(1.2-5.3)$ & $2.4(0.7-3.3)$ \\
\hline Students & 23 & 18 & 1 & 1 \\
\hline \multicolumn{5}{|c|}{ Monthly household income (ETB) } \\
\hline$<500$ & 176 & 69 & $2.8(1.1-3.8)$ & $2.5(1.5-6.3)^{*}$ \\
\hline $500-1000$ & 287 & 106 & $2.2(1.1-3.8)$ & $1.8(0.7-4.1)$ \\
\hline $1001-2000$ & 41 & 16 & $1.6(1.20-3.45)$ & $1.3(0.5-3.4)$ \\
\hline$>2000$ & 66 & 12 & 1 & 1 \\
\hline \multicolumn{5}{|c|}{ Attendance at antenatal clinic } \\
\hline Yes & 84 & 160 & 1 & 1 \\
\hline No & 188 & 143 & $2.8(2.3-5.3)$ & $4.0(3.3-9.1)^{*}$ \\
\hline \multicolumn{5}{|c|}{ Decision-maker for obstetric care } \\
\hline Woman herself & 147 & 87 & $3.0(1.8-5.0)$ & $2.1(1.2-3.8)^{*}$ \\
\hline Husband & 314 & 98 & $5.3(3.2-13.3)$ & $2.1(0.8-5.2)$ \\
\hline Neighbours & 111 & 18 & $4.4(1.1-9.5)$ & $1.8(0.5-2.2)$ \\
\hline
\end{tabular}


the healthcare facility (aOR 2.9; 95\% CI 1.3 - 6.4) than those who did visit an antenatal clinic. Compared with women who gave birth by spontaneous vaginal delivery, women who required assisted deliveries or who experienced an abortion were significantly more likely to experience a delay in service delivery $\left(\mathrm{aOR}_{\text {vacuum assistance }} 2.1 ; 95 \% \mathrm{CI} 1.03\right.$ - 4.26; $\mathrm{aOR}_{\text {abortion }} 3.5 ; 95 \% \mathrm{CI} 1.02$ - 5.32).

The likelihood of experiencing a delay in service delivery was $59 \%$ lower among women with obstetric complications $(\mathrm{aOR}=0.41$; 95\% CI 0.26 - 0.66) than among those who did not present with complications.

Table 3. Responses regarding transportation to the nearest healthcare facility for emergency care $(N=775)$

\begin{tabular}{ll}
\hline Characteristic & $\boldsymbol{n}(\%)$ \\
\hline Difficulty in accessing transport & \\
No & $541(69.8)$ \\
Yes & $234(30.2)$ \\
Mode of transport & \\
Motorised & $313(40.4)$ \\
Carried on homemade stretcher & $116(14.9)$ \\
Animal & $64(8.2)$ \\
Walking & $282(36.4)$
\end{tabular}

\section{Discussion}

In this study, $26.2 \%$ of respondents reported difficulty in seeking emergency obstetric care, resulting in a mean delay of 90 minutes. This contrasts with the results from a study from Bahir Dar in Amhara, Ethiopia, where $37.8 \%$ women reported experiencing the first maternal delay, with a mean delay of 8 hours. ${ }^{[2]}$ However, the number of women who experienced this type of delay in our study is comparable with results from a Rwandan study (22.7\%), ${ }^{[18]}$ but considerably lower than the $73.3 \%$ who reported this category of delay in a Tanzanian study. ${ }^{[19]}$

In our study, $\sim$ half of the respondents $(n=412 ; 53.2 \%)$ reported that the decision to seek obstetric care at a health facility was made by their husband. This finding likely relates to the cultural context, given that the majority of the respondents (83\%) had attended antenatal care and were expected to recognise signs of emergency better than their husbands. The number of women who did not attend an antenatal clinic during their pregnancy (17\%) is higher than what was reported in a comparable study from Tanzania (11.1\%). ${ }^{[19]}$

In the present study, maternal age, education level, monthly household income and antenatal visits were significant predictors of the first maternal delay. This is consistent with a study conducted in Bahir Dar, Ethiopia. ${ }^{[20]}$

Similar to Awoke and Seleshi, ${ }^{[20]}$ we found that a lack of accessible transport presented a notable obstacle in reaching a healthcare facility, and contributed to $30.2 \%$ of responses related to the second

Table 4. Multivariate analysis showing factors associated with a delay in reaching a healthcare facility for emergency obstetric care (maternal delay 2) among respondents $(N=775)$

\begin{tabular}{|c|c|c|c|c|}
\hline Variables & No delay experienced, $n$ & Delay experienced, $n$ & OR $(95 \% \mathrm{CI})$ & aOR $(95 \% \mathrm{CI})$ \\
\hline \multicolumn{5}{|l|}{ Age (years) } \\
\hline $15-19$ & 12 & 30 & 1 & 1 \\
\hline $20-34$ & 480 & 186 & $6.8(2.8-16.0)$ & $0.05(0.01-0.14)^{*}$ \\
\hline $35-49$ & 49 & 18 & $1.0(0.59-1.8)$ & $0.04(0.02-0.13)$ \\
\hline \multicolumn{5}{|l|}{ Occupation } \\
\hline Unemployed & 458 & 210 & $2.2(1.43-3.6)$ & $9.3(3.7-23.3)^{\star}$ \\
\hline \multicolumn{5}{|c|}{ Monthly household income (ETB) } \\
\hline$<500$ & 138 & 107 & $6.9(3.2-15.1)$ & $2.4(1.1-5.8)^{*}$ \\
\hline $500-1000$ & 301 & 92 & $2.7(1.2-5.9)$ & $0.99(0.4-2.3)$ \\
\hline $1001-2000$ & 33 & 26 & $7.8(3.1-19.1)$ & $3.3(1.2-8.9)^{*}$ \\
\hline$>2000$ & 69 & 9 & 1 & 1 \\
\hline \multicolumn{5}{|c|}{ Attendance at antenatal clinic } \\
\hline No & 71 & 60 & $2.2(1.5-3.3)$ & $1.99(1.2-3.1)^{*}$ \\
\hline \multicolumn{5}{|c|}{ Obstetric complication } \\
\hline Yes & 18 & 216 & $1.0(0.5-1.8)$ & $1.5(0.79-2.9)$ \\
\hline No & 43 & 498 & 1 & 1 \\
\hline
\end{tabular}

Table 5. Service delivery delays for emergency obstetric care at a healthcare facility (maternal delay 3 ) grouped according to outcome of pregnancy $(N=775)$

\begin{tabular}{|c|c|c|}
\hline Outcome of pregnancy & No delay experienced, $n(\%)(N=577)$ & Delay experienced, $n(\%)(N=198)$ \\
\hline Spontaneous vaginal delivery & $523(90.6)$ & $136(68.7)$ \\
\hline Abortion & $0(0.0)$ & $10(5.1)$ \\
\hline Vacuum-assisted delivery & $42(7.3)$ & $24(12.1)$ \\
\hline Perineal tear & $12(2.1)$ & $28(14.1)$ \\
\hline
\end{tabular}


Table 6. Reasons reported for a delay in receiving appropriate emergency care (maternal delay 3$)(N=198)$

\begin{tabular}{ll}
\hline Reason for delay & $\boldsymbol{n}(\%)$ \\
\hline Staff workload & $90(45.4)$ \\
Long admission process & $30(15.2)$ \\
Problem with supplies or medication & $24(12.1)$ \\
Careless staff attitude & $36(18.2)$ \\
Not mentioned & $18(9.1)$
\end{tabular}

Similar to the study in Bahir Dar, ${ }^{[20]}$ unemployment and a low monthly household income were significantly associated with the likelihood of transport-related delays in reaching a healthcare facility. However, in contrast to that study, we did not find that educational status was a significant predictor of the second maternal delay.

Approximately a quarter of respondents in our study $(n=198$; $25.5 \%)$ reported a delay in receiving emergency care once they had arrived at the healthcare facilities; a mean delay of 42.3 minutes was reported (range 10 minutes to 9 hours). However, it should be noted

Table 7. Multivariate analysis showing factors associated with a delay in service delivery related to obstetric emergencies at a healthcare facility (maternal delay 3$)$ among respondents $(N=775)$

\begin{tabular}{|c|c|c|c|c|}
\hline Variables & No delay experienced, $n$ & Delay experienced, $n$ & OR $(95 \% \mathrm{CI})$ & aOR $(95 \% \mathrm{CI})$ \\
\hline \multicolumn{5}{|l|}{ Age (years) } \\
\hline $15-19$ & 24 & 18 & $3.4(1.4-8.2)$ & $3.6(2.13-10.40)^{\star}$ \\
\hline $20-34$ & 498 & 168 & $1.5(0.80-2.9)$ & $2.9(1.1-7.1)^{\star}$ \\
\hline $35-49$ & 55 & 12 & 1 & 1 \\
\hline \multicolumn{5}{|l|}{ Marital status } \\
\hline Single & 24 & 24 & $1.0(0.28-3.5)$ & $1.7(0.16-18.1)$ \\
\hline Married & 535 & 150 & $0.28(0.08-0.88)$ & $12.3(0.95-161.0)$ \\
\hline Divorced & 12 & 18 & $1.5(0.39-5.7)$ & 0 \\
\hline Widowed & 6 & 6 & 1 & 1 \\
\hline \multicolumn{5}{|l|}{ Occupation } \\
\hline Housewife & 439 & 102 & 1 & 1 \\
\hline Government employee & 66 & 36 & $0.27(0.12-0.60)$ & $0.40(0.21-0.73)^{*}$ \\
\hline Self-employed & 60 & 36 & $0.11(0.05-0.24)$ & $0.30(0.13-0.69)^{*}$ \\
\hline Other & 12 & 24 & $0.30(0.13-0.67)$ & $0.99(0.84-1.2)$ \\
\hline \multicolumn{5}{|l|}{ Monthly household income (ETB) } \\
\hline$<500$ & 185 & 60 & 1 & 1 \\
\hline $500-1000$ & 291 & 102 & $0.95(0.46-1.9)$ & $1.1(0.72-1.8)$ \\
\hline $1001-2000$ & 47 & 12 & $1.9(0.94-3.8)$ & $1.6(0.76-3.7)$ \\
\hline$>2000$ & 54 & 24 & $1.8(0.90-3.6)$ & $0.86(0.38-1.9)$ \\
\hline \multicolumn{5}{|l|}{ Attendance at antenatal clinic } \\
\hline Yes & 479 & 65 & 1 & 1 \\
\hline No & 80 & 151 & $1.8(1.2-2.7)$ & $2.9(1.3-6.4)^{\star}$ \\
\hline \multicolumn{5}{|l|}{ Obstetric complication } \\
\hline Yes & 31 & 30 & $3.1(1.8-5.3)$ & $0.41(0.26-0.66)^{*}$ \\
\hline No & 546 & 168 & 1 & 1 \\
\hline \multicolumn{5}{|l|}{ Outcome of pregnancy } \\
\hline Spontaneous vaginal delivery & 523 & 136 & 1 & 1 \\
\hline Abortion & 2 & 8 & $0.1(0.0-0.3)$ & $3.5(1.0-5.3)^{*}$ \\
\hline Vacuum-assisted delivery & 42 & 24 & $0.1(0.0-0.1)$ & $2.1(1.0-4.2)^{\star}$ \\
\hline Perineal tear & 12 & 18 & $0.3(0.1-0.5)$ & $11.5(4.4-30.0)^{*}$ \\
\hline
\end{tabular}

maternal delay. Only $40.4 \%$ of respondents in our study used motorised transport; the remainder had to resort to using an animal (8.2\%), walking $(36.4 \%)$ or being carried on a homemade stretcher (14.9\%). Respondents travelled a minimum of 30 minutes to reach the healthcare facility, and an average of 42 minutes' walking was reported. This is notably less than travel times reported from elsewhere in in Ethiopia (3 hours) ${ }^{[20]}$ and India (2 hours or more). ${ }^{[21]}$ The difference between our finding and the other reported studies could be due partly to respondents reporting a delay in transportation as wasted time, rather than including it in the total time required to reach the healthcare facility. A study from Rwanda cited lack of transportation and the quality of roads as barriers to care. ${ }^{[18]}$ that this reported delay was the opinion of respondents and could not be confirmed from clinical notes. The proportion of women who reported a delay in service delivery is lower than what has been reported from elsewhere in Ethiopia (30.7\%) ${ }^{[20]}$ and Karachi, Pakistan $(48 \%){ }^{[10]}$ Delays in service delivery in our study are similar to those from a systematic review conducted in developing countries and the studies from Bahir Dar ${ }^{[20]}$ and Rwanda. ${ }^{[18]}$

Maternal age and occupation status were significant predictors of delayed service delivery. This contrasts with findings from elsewhere in Ethiopia, ${ }^{[20]}$ where none of the sociodemographic variables showed statistically significant association with this category of maternal delay. However, our results do support the findings from that study 
in that antenatal visits, obstetric complications and outcome of the pregnancy were significantly associated with this delay category.

The most common delay experienced by respondents in our study related to transport difficulties, followed by a delay in deciding to seek emergency care and delays related to service delivery at the healthcare facility, respectively. In contrast, delays in service delivery (maternal delay 3) were noted as the most common (79\%) in a study conducted in a public-sector tertiary teaching hospital in Ethiopia; delays related to deciding to seek care and access to facilities were less common. ${ }^{[10,22]}$

\section{Conclusion}

Our study revealed that many women experienced delays with regard to emergency obstetric care. The 'Three delays' model provides a valuable schematic framework for investigating the factors that could contribute to maternal morbidity or mortality. Reducing these delays will require a comprehensive approach, including improving the decision-making capacity of women, transportation access and equipping health facilities with the necessary resources. All factors, including cultural norms that may prevent pregnant women advocating for themselves in health issues, must be addressed.

\section{Acknowledgements. None.}

Author contributions. YW contributed to study conceptualisation and design, data analysis and interpretation, and preparing the manuscript. BD, MB and $\mathrm{MH}$ contributed to critical revision and final approval of the manuscript.

Funding. Financial assistance received from the Research and Publication directorate, Arsi University, is acknowledged.

Conflicts of interest. None.

1. Ganchimeg T, Ota E, Morisaki N, et al. Pregnancy and childbirth outcomes among adolescen mothers: A World Health Organization multicountry study. BJOG 2014;121(Suppl 1):40-48. https:// doi.org/10.1111/1471-0528.12630

2. Worku AG, Yalew AW, Afework MF. Maternal complications and women's behavior in seeking care from skilled providers in North Gondar, Ethiopia. PLoS One 2013:8(3):e60171. https://doi. org/10.1371/journal.pone.0060171

3. Ramos S, Karolinski A, Romero M, Mercer R, Maternal Mortality in Argentina Study Group. A comprehensive assessment of maternal deaths in Argentina: Translating multicentre collaborative research into action. Bull World Health Organ 2007;85(8):615-622. https://doi.org/10.2471/ blt.06.032334
4. Tessema GA, Laurence CO, Melaku YA, et al. Trends and causes of maternal mortality in Ethiopia during 1990-2013: Findings from the Global Burden of Diseases study 2013. BMC Public Health 2017;17(1):160. https://doi.org/.org/10.1186/s12889-017-4071-8

5. Pacagnella RC, Cecatti JG, Osis MJ, Souza JP. The role of delays in severe maternal morbidity and mortality: Expanding the conceptual framework. Reprod Health Matters 2012;20(39):155-163. https://doi.org/10.1016/S0968-8080(12)39601-8

6. Thaddeus S, Maine D. Too far to walk: Maternal mortality in context. Soc Sci Med 1994;38(8):10911110. https://doi.org/10.1016/0277-9536(94)90226-7

7. Barnes-Josiah D, Myntti C, Augustin A. The "three delays" as a framework for examining maternal mortality in Haiti. Soc Sci Med 1998;46(8):981-993. https://doi.org/10.1016/s0277-9536(97)10018-1

8. Mgawadere F, Unkels R, Kazembe A, Van den Broek N. Factors associated with maternal mortality in Malawi: Application of the three delays model. BMC Pregnancy Childbirth 2017;17(1):219. https://doi.org/10.1186/s12884-017-1406-5

9. Alkema L, Chou D, Hogan D, et al. Global, regional, and national levels and trends in materna mortality between 1990 and 2015, with scenario-based projections to 2030: A systematic analysis by the UN Maternal Mortality Estimation Inter-Agency Group. Lancet 2016;387(10017):462-474.

10. Shah N, Hossain N, Shoaib R, Hussain A, Gillani R, Khan NH. Socio-demographic characteristics and the three delays of maternal mortality. J Coll Physicians Surg Pak 2009;19(2):95-98. https://doi org/: 02.2009/JCPSP.9598

11. Calvello EJ, Skog AP, Tenner AG, Wallis LA. Applying the lessons of maternal mortality reduction to global emergency health. Bull World Health Organ 2015;93(6):417-423. https://doi.org/10.2471/ BLT.14.146571

12. Knight HE, Self A, Kennedy SH. Why are women dying when they reach hospital on time? A systematic review of the 'third delay'. PLoS One 2013;8(5):e63846. https://doi.org/10.1371/journal. pone.0063846

13. Syed U, Khadka N, Khan A, Wall S. Care-seeking practices in South Asia: Using formative research to design program interventions to save newborn lives. J Perinatol 2008;28(Suppl 2):S9-13. https:// doi.org/10.1038/jp.2008.165

14. Soma-Pillay P, Pattinson RC. Barriers to obstetric care among maternal near misses. S Afr Med J 2016;106(11):1110-1113. https://doi.org/10.7196/SAMJ.2016.v106i11.10726

15. Koblinsky M. Reducing maternal and perinatal mortality through a community collaborative approach: Introduction to a special issue on the Maternal and Newborn Health in Ethiopia Partnership (MaNHEP). J Midwifery Womens Health 2014;59(Suppl 1):S1-5. https://doi. org/10.1111/jmwh.12174

16. Goodman DM, Srofenyoh EK, Ramaswamy R, et al. Addressing the third delay: Implementing a novel obstetric triage system in Ghana. BMJ Glob Health 2018;3(2):e000623. https://doi. org/10.1136/bmigh-2017-000623

17. Austin A, Langer A, Salam RA, Lassi ZS, Das JK, Bhutta ZA. Approaches to improve the quality of maternal and newborn health care: An overview of the evidence. Reprod Health 2014;11(Suppl 2):S1. https://doi.org/10.1186/1742-4755-11-S2-S1

18. Benimana C, Small M, Rulisa S. Preventability of maternal near miss and mortality in Rwanda: A case series from the University Teaching Hospital of Kigali (CHUK). PLoS One 2018;13(6):e0195711. https://doi.org/.org/10.1371/journal.pone.0195711

19. Pembe AB, Paulo C, D’mello BS, Van Roosmalen J. Maternal mortality at Muhimbili National Hospital in Dar-es-Salaam, Tanzania in the year 2011. BMC Pregnancy Childbirth 2014;14(1):320. https://doi.org/10.1186/1471-2393-14-320

20. Awoke W, Seleshi K. Maternal delays in utilizing institutional delivery services, Bahir Dar, Ethiopia. Health 2013;5(6):1026-1031. https://doi.org/10.4236/health.2013.56137

21. Khan N, Pradhan MR. Identifying factors associated with maternal deaths in Jharkhand, India: A verbal autopsy study. J Health Popul Nutr 2013;31(2):262-271. https://doi.org/10.3329/jhpn. v31i2.16391

22. Tita AT, Selwyn BJ, Waller DK, Kapadia AS, Dongmo S. Evidence-based reproductive health care in Cameroon: Population-based study of awareness, use and barriers. Bull World Health Organ 2005:83(12):895-903.

Accepted 16 September 2019 